\title{
Throwing Motion Control of the Pendubot and Instability Analysis of the Zero Dynamics
}

\author{
Takuya Shoji, Kazuma Sekiguchi, and Mitsuji Sampei
}

\begin{abstract}
This paper presents systematic methods for throwing motion control of the Pendubot based on the concept of explosively unstable zero dynamics. The Pendubot, an underactuated two-link planar robot, is investigated as a dynamic model of the superior limbs for imitation of human throwing motion whose models are fundamentally underactuated in nature. The controller is designed based on input-output linearization and output zeroing control since the Pendubot is not input-state linearizable. The originality of this paper is to intentionally destabilize the zero dynamics, a nonlinear dynamics which remains unobservable from the output when the partially linearized dynamics converges exponentially to zero, to generate dynamic acceleration of the ball. Mathematical analysis of ordinary differential equations guarantees the explosive instability of the zero dynamics. Numerical simulations verify the effectiveness of the proposed control strategy.
\end{abstract}

\section{INTRODUCTION}

Control problems of underactuated mechanical systems have attracted a number of researchers in the field of control theory and robotics since it consists of both pure and applied research interests. Westervelt et al. [1] presented systematic methods for achieving stable, agile, and efficient locomotion in bipedal robots. Anton et al. [2] and Freidovich et al. [3] proposed a new constructive tool for orbital stabilization of underactuated nonlinear systems based on the concept of virtual holonomic constraints. Mettin et al. [4] proposed a new approach for solving an optimal control problem of ball pitching with an underactuated model of a human arm. Katsumata et al. [5], Ichinose et al. [6], and Shoji et al. [7] claimed that the unstable zero dynamics generated dynamic acceleration of a ball when the input is devoted to constrain the ball on a geometric path via input-output linearization and output zeroing control. The major omission of the previous works, however, was the lack of zero dynamics analysis. The zero dynamics was too complicated to calculate a general solution of the differential equation and to give exact analysis since the output consisted of nonlinear functions with respect to the configuration variables. Moreover, a torsion spring mounted on the passive joint of the Pendubot might help the controller to produce the required torque for throwing motion control. Therefore instability of the zero dynamics was not guaranteed.

T. Shoji is with the Department of Mechanical and Control Engineering, Tokyo Institute of Technology, 2-12-1 Ookayama, Meguro-ku, Tokyo 1528550, Japan shoji@sc.ctrl.titech.ac.jp

K. Sekiguchi is with the Department of Mechanical and Control Engineering, Tokyo Institute of Technology, 2-12-1 Ookayama, Meguro-ku, Tokyo 152-8550, Japan sekiguchi@ctrl.titech.ac.jp

M. Sampei is with the Department of Mechanical and Control Engineering, Tokyo Institute of Technology, 2-12-1 Ookayama, Meguro-ku, Tokyo 152-8550, Japan sampei@ctrl.titech.ac.jp
The purpose of this paper is to present systematic methods for throwing motion control of the Pendubot based on the concept of explosively unstable zero dynamics and to give zero dynamics analysis. The controller design is based on input-output linearization and output zeroing control since the control objective is motion generation of underactuated mechanical systems which can never be achieved by approximate linearization nor trajectory tracking control. The output in this paper is designed to consist of linear functions with respect to the configuration variables while the output in the previous work consisted of nonlinear functions with respect to the configuration variables, which made it difficult to give zero dynamics analysis.

This paper is organized as follows: In Section II, a dynamic model is constructed with the Pendubot. In Section III, control strategy for throwing motion is proposed based on the concept of explosively unstable zero dynamics via inputoutput linearization and output zeroing control. In Section IV, instability of the zero dynamics is discussed based on second order ordinary differential equations. In Section $\mathrm{V}$, numerical simulations confirm the effectiveness of the proposed control strategy. Section VI concludes the paper.

\section{DynAmic Model And State Equation}

This section introduces a dynamic model for throwing motion control of the Pendubot as shown in Fig. 1. The Pendubot is an underactuated two-link planar robot with an actuator at joint 1 but no actuator at joint 2, consisting of links 1 and 2 which represent the upper arm and the forearm, respectively. The end-effector holds a ball and releases it instantaneously when it enters the first quadrant for the first time while its center of mass rotates in a positive direction around joint 1 . The notation is fixed as follows: For $i=1,2$, $m_{i}$ denotes the mass of link $i$; $l_{i}$ denotes the length of link $i$; $l_{g i}$ denotes the distance from the previous joint to the center of mass of link $i ; J_{i}$ denotes the moment of inertia of link $i$ about an axis coming out of the page, passing through the center of mass of link $i$; and $m_{b}$ denotes the mass of the ball.

Dynamics of the Pendubot can be described by the system of two nonlinear differential equations, originating from Lagrangian mechanics

$$
M(q) \ddot{q}+C(q, \dot{q}) \dot{q}+G(q)=B u
$$

with the vector of generalized coordinates

$$
q:=\left[\begin{array}{ll}
\theta_{1} & \theta_{2}
\end{array}\right]^{T}
$$


the inertia matrix

$$
M(q)=\left[\begin{array}{cc}
p_{1}+p_{3}+2 p_{2} \cos \theta_{2} & p_{3}+p_{2} \cos \theta_{2} \\
p_{3}+p_{2} \cos \theta_{2} & p_{3}
\end{array}\right],
$$

the matrix corresponding to Coriolis and centrifugal forces

$$
C(q, \dot{q})=\left[\begin{array}{cc}
-p_{2} \dot{\theta}_{2} \sin \theta_{2} & -p_{2}\left(\dot{\theta}_{1}+\dot{\theta}_{2}\right) \sin \theta_{2} \\
p_{2} \dot{\theta}_{1} \sin \theta_{2} & 0
\end{array}\right],
$$

the gravity vector

$$
G(q)=\left[\begin{array}{c}
g p_{4} \cos \theta_{1}+g p_{5} \cos \left(\theta_{1}+\theta_{2}\right) \\
g p_{5} \cos \left(\theta_{1}+\theta_{2}\right)
\end{array}\right],
$$

and the input matrix mapping the applied torque to generalized forces

$$
B=\left[\begin{array}{ll}
1 & 0
\end{array}\right]^{T}
$$

where $g$ is the acceleration due to gravity; $\theta_{1}$ is the absolute angle of the first link; $\theta_{2}$ is the relative angle between the two links; $\tau_{1}$ is the controlled torque, applied to the first link. Physical parameter is combined to

$$
\begin{aligned}
& p_{1}=I_{1}+l_{g 1}{ }^{2} m_{1}+l_{1}^{2}\left(m_{2}+m_{b}\right), \\
& p_{2}=l_{1}\left(l_{g 2} m_{2}+l_{2} m_{b}\right), \\
& p_{3}=I_{2}+l_{g 2}{ }^{2} m_{2}+l_{2}^{2} m_{b}, \\
& p_{4}=l_{g 1} m_{1}+l_{1}\left(m_{2}+m_{b}\right), \\
& p_{5}=l_{g 2} m_{2}+l_{2} m_{b} .
\end{aligned}
$$

The dynamic model of the Pendubot is written in state space form by defining

$$
\begin{aligned}
\dot{x} & =\left[\begin{array}{c}
\dot{q} \\
M(q)^{-1}[-C(q, \dot{q}) \dot{q}-G(q)+B u]
\end{array}\right] \\
& =: f(x)+g(x) u,
\end{aligned}
$$

with the state vector

$$
x:=\left[\begin{array}{ll}
q^{T} & \dot{q}^{T}
\end{array}\right]^{T}
$$

and the input vector

$$
u:=\tau_{1}
$$

\section{Controller Design}

This section proposes control strategy for throwing motion of the Pendubot. Since the dynamic model is not input-state linearizable, input-output linearization decouples the system into linear and nonlinear dynamics. The originality of this paper is to intentionally destabilize the zero dynamics, a nonlinear dynamics which remains unobservable from the output when the partially linearized dynamics converges exponentially to zero via output zeroing control. Dynamic acceleration of the ball is then generated by obtaining energy from divergence of the unstable zero dynamics.

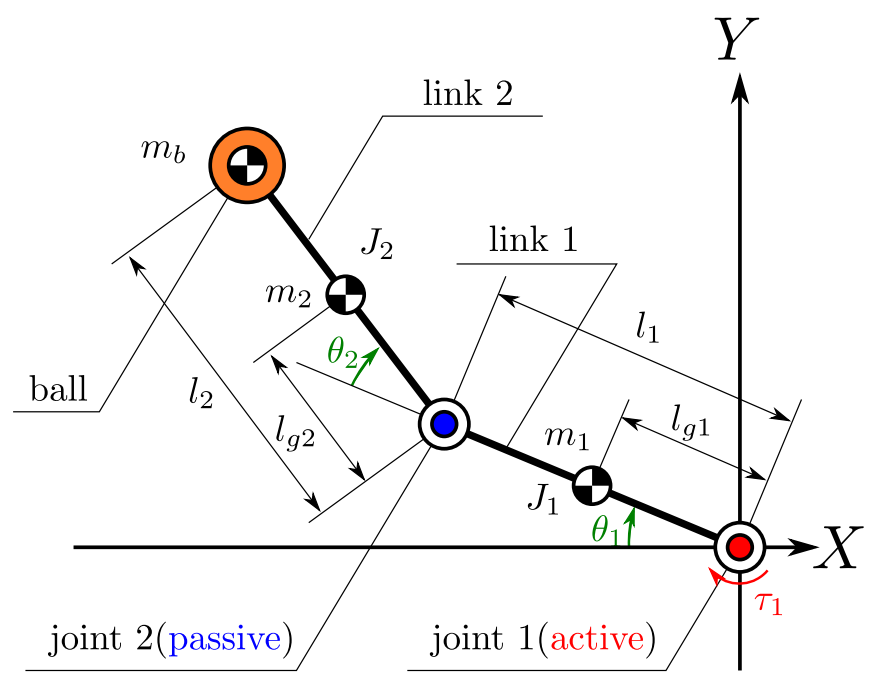

Fig. 1. Dynamic model of the Pendubot.

\section{A. Input-Output Linearization}

For the underactuation of the dynamic model which is not input-state linearizable, input-output linearization is considered with an output

$$
y=h(x):=\theta_{2}-\theta_{2 d} \in \mathbb{R},
$$

where $\theta_{2 d}$ denotes the desired angle of joint 2 . Note that $y=0$ means $\theta_{2}=\theta_{2 d}$. Since the designed output depends only on the configuration variables, then, due to the second order nature of the robot model (1), the first time derivative of the output along solutions of the state equation

$$
\dot{y}=\frac{d y}{d t}=\frac{\partial h(x)}{\partial x} \dot{x}=L_{f} h(x)
$$

does not depend directly on the input. Hence, the relative degree of the output is at least two. The second time derivative of the output along solutions of the state equation is derived as

$$
\ddot{y}=\frac{d^{2} y}{d t^{2}}=\frac{\partial L_{f} h(x)}{\partial x} \dot{x}=L_{f}^{2} h(x)+L_{g} L_{f} h(x) u,
$$

which indicates that the relative degree of the output is two. The preliminary feedback

$$
u=L_{g} L_{f} h(x)^{-1}\left(v-L_{f}^{2} h(x)\right)
$$

yields the output dynamics $\ddot{y}=v$ when $v$ is a new input. The dynamics of the Pendubot is then partially linearized via input-output linearization as

$$
\frac{d}{d t}\left[\begin{array}{l}
y \\
\dot{y}
\end{array}\right]=\left[\begin{array}{ll}
0 & 1 \\
0 & 0
\end{array}\right]\left[\begin{array}{l}
y \\
\dot{y}
\end{array}\right]+\left[\begin{array}{l}
0 \\
1
\end{array}\right] v .
$$

This differential equation called the external dynamics, which gives a linear relationship between the input and the output. Since the relative degree of the output is two and is lower than the dimension of the original system, only the external dynamics of dimension two is linearized. A 
nonlinear dynamics, called the internal dynamics, remains unobservable from the output and is given by

$$
\frac{d}{d t}\left[\begin{array}{l}
z \\
\dot{z}
\end{array}\right]=\left[\begin{array}{c}
\dot{z} \\
-\frac{\beta(y)}{\alpha(y)} \dot{z}^{2}-\frac{\gamma(y, z)}{\alpha(y)}
\end{array}\right]+\left[\begin{array}{c}
0 \\
-\frac{p_{3}}{\alpha(y)}
\end{array}\right] v
$$

where

$$
\begin{aligned}
\alpha(y) & =p_{3}+p_{2} \cos \left(y+\theta_{2 d}\right), \\
\beta(y) & =p_{2} \sin \left(y+\theta_{2 d}\right), \\
\gamma(y, z) & =p_{5} g \cos \left(y+z+\theta_{2 d}\right) .
\end{aligned}
$$

The new coordinate of the internal dynamics as a counterpart of the output

$$
z:=\theta_{1} \in \mathbb{R}^{1}
$$

defines a mapping

$$
\Phi(x):=\left[\begin{array}{c}
y \\
\dot{y} \\
z \\
\dot{z}
\end{array}\right]=\left[\begin{array}{c}
\theta_{2}-\theta_{2 d} \\
\dot{\theta}_{2} \\
\theta_{1} \\
\dot{\theta}_{1}
\end{array}\right] \in \mathbb{R}^{4}
$$

whose jacobian matrix

$$
\frac{\partial \Phi(x)}{\partial x}=\left[\begin{array}{llll}
0 & 1 & 0 & 0 \\
0 & 0 & 0 & 1 \\
1 & 0 & 0 & 0 \\
0 & 0 & 1 & 0
\end{array}\right]
$$

is nonsingular for all $x$ as a global coordinates transformation. Note that gravity terms are all rearranged in $\gamma(y, z)$.

\section{B. Output Zeroing Control}

For the linearity of the external dynamics of dimension two, the state variable feedback

$$
v=-K_{P} y-K_{D} \dot{y}
$$

results in

$$
\ddot{y}+K_{D} \dot{y}+K_{P} y=0,
$$

that is

$$
\frac{d}{d t}\left[\begin{array}{c}
y \\
\dot{y}
\end{array}\right]=\left[\begin{array}{cc}
0 & 1 \\
-K_{P} & -K_{D}
\end{array}\right]\left[\begin{array}{l}
y \\
\dot{y}
\end{array}\right] .
$$

For $K_{P}, K_{D}>0$, the external dynamics converges exponentially to zero. For $y \equiv 0$, that is $\theta_{2} \equiv \theta_{2 d}$, the system is represented by a reduced order dynamics called the zero dynamics,

$$
\frac{d}{d t}\left[\begin{array}{c}
z \\
\dot{z}
\end{array}\right]=\left[\begin{array}{c}
\dot{z} \\
-\frac{\beta(0)}{\alpha(0)} \dot{z}^{2}-\frac{\gamma(0, z)}{\alpha(0)}
\end{array}\right],
$$

provided that initial conditions are chosen to satisfy the constraint. Note that $\alpha(0)$ and $\beta(0)$ are constants and $\gamma(0, z)$ is a function with respect to $z=\theta_{1}$, the angle of joint 1 .

\section{ZERO DYNAMICS ANALYSIS}

This section analyzes instability of the zero dynamics derived in the previous section. The instability analysis is based on solving second order ordinary differential equations. A general solution of the zero dynamics is derived to reveal the effects of changes in initial conditions on level sets. Note that this section contains main results of this paper.

\section{A. Formulation of Zero Dynamics}

The differential equation of the zero dynamics defined in the previous section can also be formulated as a second order ordinary differential equation,

$$
\alpha(0) \ddot{z}+\beta(0) \dot{z}^{2}+\gamma(0, z)=0,
$$

by substituting constraint $y=0$, that is $\theta_{2}=\theta_{2 d}$, into nonholonomic constraint of the dynamic model where

$$
\begin{aligned}
\alpha(0) & =p_{3}+p_{2} \cos \theta_{2 d}=\text { Const. } \\
\beta(0) & =p_{2} \sin \theta_{2 d}=\text { Const. } \\
\gamma(0, z) & =p_{5} g \cos \left(z+\theta_{2 d}\right) .
\end{aligned}
$$

Lemma 1: Physical parameter of the Pendubot satisfies $\alpha(0)>0$ to avoid the existence of unconsidered singular points, which results in $2 l_{2} \geq 3 l_{1}$ when link 2 is assumed to be $J_{2}=m_{2} l_{2}^{2} / 12$ and $l_{g 2}=l_{2} / 2$.

Proof: The proof is omitted for simple calculation.

Lemma 2: $\gamma(0, z)$ given by (35) is bounded by a positive constant $D_{\gamma}$ as $|\gamma(0, z)| \leq D_{\gamma}$ for every $z \in \mathbb{R}$.

Proof: The proof is omitted for simple calculation.

With the aid of these lemmas, explosively instability of the zero dynamics can be discussed as follows.

\section{B. Explosive Instability of Zero Dynamics}

The section shows explosive instability of the zero dynamics and reveals mathematical conditions for the zero dynamics to be destabilized by output zeroing control. The first step is to define instability and explosive instability for a solution of the differential equation.

Definition 1: Consider an ordinary differential equation

$$
\dot{z}=f(z), \quad z \in \mathbb{R}^{n}
$$

where $f(z)$ is a vector field in $\mathbb{R}^{n}$. A solution $z(t)$ is unstable if the solution escapes to infinity in infinite time as $\lim _{t \rightarrow \infty}\|z(t)\|=\infty$. Moreover, a solution $z(t)$ is explosively unstable if the solution escapes to infinity in finite time as $\lim _{t \rightarrow T}\|z(t)\|=\infty$ where $T<\infty . \triangleleft$

Remark 1: This paper defines instability for a solution of the differential equation to introduce the concept of blow-up phenomena in finite escape time while instability is generally defined for an equilibrium point. $\triangleleft$

Theorem 1: Consider the zero dynamics given by a second order ordinary differential equation

$$
\alpha(0) \ddot{z}+\beta(0) \dot{z}^{2}+\gamma(0, z)=0,
$$

where $\alpha(0)>0$ and $|\gamma(0, z)| \leq D_{\gamma}$ for every $z \in \mathbb{R}$. If $\beta(0)<$ 0 for all $t \in \mathbb{R}^{+}$, then a solution $z(t)$ with initial conditions $\left|\dot{z}_{0}\right|>\sqrt{D_{\gamma} /(-\beta(0))}$ is explosively unstable and the solution escapes to positive infinity in finite time.

Proof: Due to space constraints, only a sketch of the proof is given in the following. Substituting the differential equation (37) into $|\gamma(0, z)| \leq D_{\gamma}$ gives

$$
\left|\alpha(0) \ddot{z}+\beta(0) \dot{z}^{2}\right| \leq D_{\gamma}
$$


indicating that a solution of the differential equation should have upper and lower bounds. Since $\beta(0)<0$ for all $t \in \mathbb{R}^{+}$, the upper bound

$$
\alpha(0) \ddot{z}+\beta(0) \dot{z}^{2} \leq D_{\gamma}
$$

is valid for all $\dot{z} \in \mathbb{R}$ while the lower bound

$$
-D_{\gamma} \leq \alpha(0) \ddot{z}+\beta(0) \dot{z}^{2}
$$

is divided by $\dot{z}$ into three discrete intervals as follows: For $|\dot{z}|>\sqrt{D_{\gamma} /(-\beta(0))}$, a solution of the differential equation is upper and lower bounded by

$$
\lambda_{f t}(t) \leq \dot{z}(t) \leq \lambda_{f t}(t), \kappa_{f t}(t) \leq z(t) \leq \kappa_{f t}(t),
$$

where $\lambda_{f t}(t)$ and $\kappa_{f t}(t)$ are some functions with respect to $t$ which escape to positive infinity in finite time. For $|\dot{z}|<\sqrt{D_{\gamma} /(-\beta(0))}$, a solution of the differential equation is upper and lower bounded by

$$
\lambda_{i t}(t) \leq \dot{z}(t) \leq \lambda_{f t}(t), \kappa_{i t}(t) \leq z(t) \leq \kappa_{f t}(t),
$$

where $\lambda_{i t}(t)$ and $\kappa_{i t}(t)$ are some functions with respect to $t$ that exist for infinite time. Therefore, a solution of the zero dynamics with initial conditions $\left|\dot{z}_{0}\right|>\sqrt{D_{\gamma} /(-\beta(0))}$ is explosively unstable and the solution escapes to positive infinity in finite time. This completes the proof.

Remark 2: The opposite of Theorem 1 is equally true. If $\beta(0)>0$ for all $t \in \mathbb{R}^{+}$, then a solution $z(t)$ with initial conditions $\left|\dot{z}_{0}\right|>\sqrt{D_{\gamma} / \beta(0)}$ is explosively unstable and the solution escapes to negative infinity in finite time. $\triangleleft$

\section{General Solution of Zero Dynamics}

A general solution of the zero dynamics governed by the differential equation (32) is derived as

$$
\begin{aligned}
I(z, \dot{z}) & :=\left(\dot{z}^{2}+\frac{2 p_{5} g \sin (z+\psi)}{\sqrt{\alpha^{2}(0)+4 \beta^{2}(0)}}\right) e^{\frac{2 \beta(0)}{\alpha(0)} z} \\
& =\left(\dot{z}_{0}^{2}+\frac{2 p_{5} g \sin \left(z_{0}+\psi\right)}{\sqrt{\alpha^{2}(0)+4 \beta^{2}(0)}}\right) e^{\frac{2 \beta(0)}{\alpha(0)} z_{0}} \\
& =I\left(z_{0}, \dot{z}_{0}\right)=: I_{0}=\text { Const. }
\end{aligned}
$$

where

$$
\psi=\tan ^{-1} \frac{\alpha(0) \sin \theta_{2 d}+2 \beta(0) \cos \theta_{2 d}}{\alpha(0) \cos \theta_{2 d}-2 \beta(0) \sin \theta_{2 d}} .
$$

Procedure for deriving the general solution is shown in the following.

1) variable transformation,

$$
w=\frac{1}{2} \dot{z}^{2} .
$$

2) chain rule,

$$
\ddot{z}=\frac{d}{d t}\left(\frac{d z}{d t}\right)=\frac{d z}{d t} \frac{d \dot{z}}{d z}=\frac{d w}{d z} .
$$

3) nonhomogeneous linear differential equation,

$$
\alpha(0) \frac{d w}{d z}+2 \beta(0) w=-\gamma(0, z) .
$$

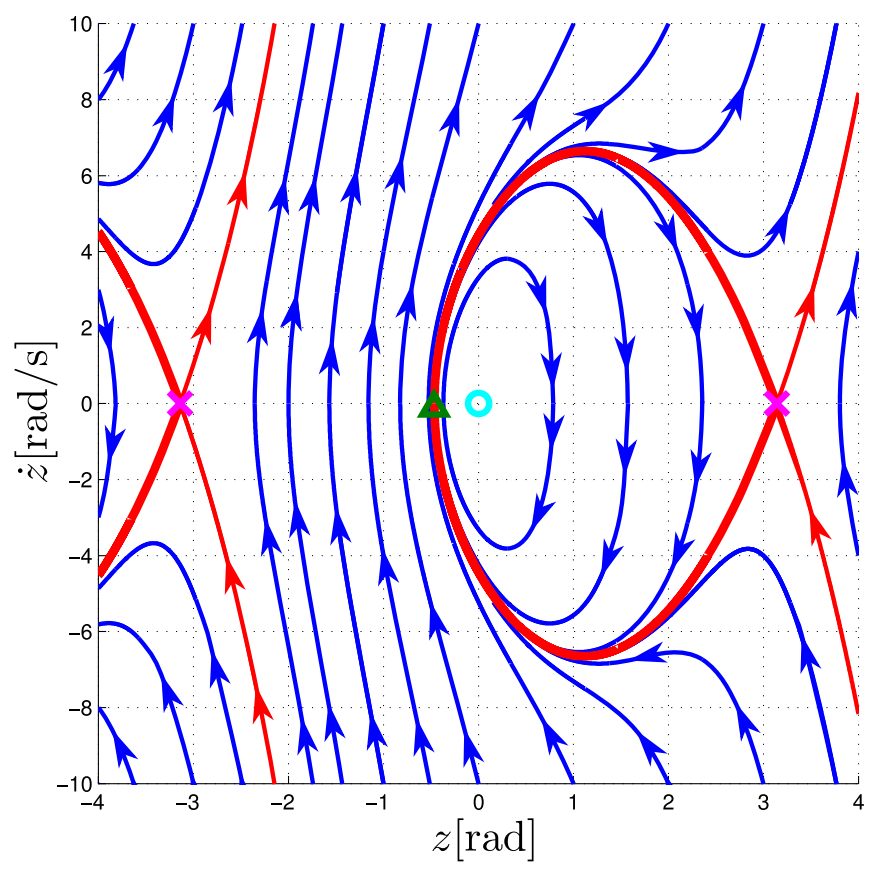

Fig. 2. Integral curve of the zero dynamics.

4) integration for general solution,

$$
\begin{aligned}
& \left(w+\frac{p_{5} g \sin (z+\psi)}{\sqrt{\alpha^{2}(0)+4 \beta^{2}(0)}}\right) e^{\frac{2 \beta(0)}{\alpha(0)} z} \\
& =\left(w_{0}+\frac{p_{5} g \sin \left(z_{0}+\psi\right)}{\sqrt{\alpha^{2}(0)+4 \beta^{2}(0)}}\right) e^{\frac{2 \beta(0)}{\alpha(0)} z_{0}} .
\end{aligned}
$$

\section{Equilibrium and Representative Points}

Fig. 2 shows integral curve of the zero dynamics. Since gravity terms of the zero dynamics and the original system are both rearranged in $\gamma(0, \dot{z})$ and $\gamma(y, \dot{z})$, respectively, the equilibrium points which satisfy

$$
\gamma(0, \dot{z}):=p_{5} g \cos \left(z+\theta_{2 d}\right)=0
$$

can be derived as follows:

$\times z_{s}$ (saddles), where the absolute angle of link 2 given by $\theta_{1}+\theta_{2}=: z+\theta_{2}$ satisfies

$$
z_{s}=\frac{\pi}{2}+2 n \pi-\theta_{2 d},
$$

$z_{c}$ (centers), where the absolute angle of link 2 given by $\theta_{1}+\theta_{2}=: z+\theta_{2}$ satisfies

$$
z_{c}=-\frac{\pi}{2}+2 n \pi-\theta_{2 d}
$$

$z_{r}$ (representative points), where the absolute angle of link 1 given by $\theta_{1}=: z_{r}$ satisfies

$$
\sin \left(z_{r}+\psi\right) e^{\frac{2 \beta(0)}{\alpha(0)} z_{r}}=\frac{\alpha(0) e^{\frac{2 \beta(0)}{\alpha(0)}\left(\frac{\pi}{2}+2 n \pi-\theta_{2 d}\right)}}{2 \sqrt{\alpha^{2}(0)+4 \beta^{2}(0)}} .
$$

This equation is called the transcendental function, which cannot be solved with respect to $z_{r}$. The numerical solution of the equation, however, can be derived 
as

$$
\theta_{1}=-0.463978+2 n \pi[\mathrm{rad}]
$$

provided that physical parameter is taken from Table I and the desired angle of joint 2 is fixed as $\theta_{2 d}=-\pi / 2$ for numerical computation.

Note that changes in $\theta_{2 d}$ result in moving positions of equilibrium and representative points.

Theorem 2: Consider the zero dynamics given by a second order ordinary differential equation (37), where $\alpha(0)>0$ and $|\gamma(0, z)| \geq D_{\gamma}$ for every $z \in \mathbb{R}$. Suppose that a general solution of the differential equation (37) is derived as

$$
\begin{aligned}
I(z, \dot{z}) & =\left(\dot{z}^{2}+\frac{2 p_{5} g \sin (z+\psi)}{\sqrt{\alpha^{2}(0)+4 \beta^{2}(0)}}\right) e^{\frac{2 \beta(0)}{\alpha(0)} z} \\
& =\left(\dot{z}_{0}^{2}+\frac{2 p_{5} g \sin \left(z_{0}+\psi\right)}{\sqrt{\alpha^{2}(0)+4 \beta^{2}(0)}}\right) e^{\frac{2 \beta(0)}{\alpha(0)} z_{0}} \\
& =I\left(z_{0}, \dot{z}_{0}\right)=: I_{0}=\text { Const. }
\end{aligned}
$$

where

$$
\psi=\tan ^{-1} \frac{\alpha(0) \sin \theta_{2 d}+2 \beta(0) \cos \theta_{2 d}}{\alpha(0) \cos \theta_{2 d}-2 \beta(0) \sin \theta_{2 d}} .
$$

Note that fixing initial conditions $z_{0}, \dot{z}_{0}$ corresponds to choosing a level set $I_{0}$ of the general solution of the zero dynamics. If the initial level set is chosen to satisfy

$$
I\left(z_{s}(n), 0\right) \leq I_{0} \leq I\left(z_{s}(n-1), 0\right)
$$

where

$$
z_{s}(n)=\frac{\pi}{2}+2 n \pi-\theta_{2 d}
$$

with $\beta(0)<0$ for all $t \in \mathbb{R}^{+}$, then a solution of the zero dynamics with initial conditions

$$
z_{s}(n) \leq z_{0} \leq z_{r}(n),
$$

is explosively unstable and the solution escapes to positive infinity in finite time where $z_{r}(n)$ denotes the corresponding representative point.

Proof: Theorem 1 shows that a solution of (37) with initial conditions $\left|\dot{z}_{0}\right|>\sqrt{D_{\gamma} /(-\beta(0))}$ is explosively unstable and the solution escapes to positive infinity in finite time, where $D_{\gamma}$ is a positive constant which bounds $|\gamma(0, z)| \leq D_{\gamma}$. Since the partial derivative of level set $I(z, \dot{z})$ with respect to z,

$$
\begin{aligned}
\frac{\partial I(z, \dot{z})}{\partial z}= & \left(\frac{2 \beta(0)}{\alpha(0)} \dot{z}^{2}+\frac{2 \beta(0)}{\alpha(0)} \frac{2 p_{5} g \sin (z+\psi)}{\sqrt{\alpha^{2}(0)+4 \beta^{2}(0)}}\right. \\
& \left.+\frac{2 p_{5} g \cos (z+\psi)}{\sqrt{\alpha^{2}(0)+4 \beta^{2}(0)}}\right) e^{\frac{2 \beta(0)}{\alpha(0)} z},
\end{aligned}
$$

is a linear combination of a quadratic function with respect to $\dot{z}$ and sine and cosine functions with respect to $z$, which results in $\partial I(z, \dot{z}) / \partial z<0$ for arbitrarily large $\dot{z}$ when $\beta(0)<$ 0 for all $t \in \mathbb{R}^{+}$. Moreover, positions of the equilibrium points are derived as $z=\pi / 2+n \pi-\theta_{2 d}$. By the divergence theorem, therefore, a solution of the zero dynamics which passes through the domain (58) is explosively unstable and the solution escapes to positive infinity in finite time with the initial level set chosen from $(56)$ when $\beta(0)<0$ for all $t \in \mathbb{R}^{+}$. This completes the proof.

Remark 3: The opposite of Theorem 2 is equally true when $\beta(0)>0$ for all $t \in \mathbb{R}^{+} . \triangleleft$

\section{E. Generalization of Zero Dynamics Analysis}

The purpose of this section is to extend Theorem 1 for the generalized zero dynamics, which widely covers other mechanical systems.

Theorem 3: Consider the zero dynamics given by a second order ordinary differential equation

$$
\alpha(z) \ddot{z}+\beta(z) \dot{z}^{2}+\gamma(z)=0,
$$

where $0<d_{\alpha} \leq \alpha(z) \leq D_{\alpha}$ and $|\gamma(z)| \leq D_{\gamma}$ for every $z \in \mathbb{R}$. If $\beta(z)$ is bounded by positive constants $D_{\beta}, d_{\beta}$ as $-D_{\beta} \leq$ $\beta(z) \leq-d_{\beta}<0$ for every $z \in \mathbb{R}$, then a solution of $z(t)$ with initial conditions $\left|\dot{z}_{0}\right|>\sqrt{D_{\gamma} / d_{\beta}}$ is explosively unstable and the solution escapes to positive infinity in finite time.

Proof: Due to space constraints, only a sketch of the proof is given in the following. Substituting the differential equation (60) into $|\gamma(0, z)| \leq D_{\gamma}$ gives

$$
\frac{d_{\beta} \dot{z}^{2}-D_{\gamma}}{D_{\alpha}} \leq \ddot{z} \leq \frac{D_{\beta} \dot{z}^{2}+D_{\gamma}}{d_{\alpha}},
$$

indicating that a solution of the differential equation should have upper and lower bounds. Since $d_{\alpha}, D_{\beta}, D_{\gamma}>0$ for all $t \in \mathbb{R}^{+}$, the upper bound

$$
\ddot{z} \leq \frac{D_{\beta} \dot{z}^{2}+D_{\gamma}}{d_{\alpha}}
$$

is valid for all $\dot{z} \in \mathbb{R}$ while the lower bound

$$
\frac{d_{\beta} \dot{z}^{2}-D_{\gamma}}{D_{\alpha}} \leq \ddot{z}
$$

is divided by $\dot{z}$ into three discrete intervals as follows: For $\left|\dot{z}_{0}\right|>\sqrt{D_{\gamma} / d_{\beta}}$, a solution of the differential equation is upper and lower bounded by

$$
\lambda_{f t}(t) \leq \dot{z}(t) \leq \lambda_{f t}(t), \kappa_{f t}(t) \leq z(t) \leq \kappa_{f t}(t),
$$

where $\lambda_{f t}(t)$ and $\kappa_{f t}(t)$ are some functions with respect to $t$ that escape to positive infinity in finite time. For $|\dot{z}|<$ $\sqrt{D_{\gamma} / d_{\beta}}$, a solution of the differential equation is upper and lower bounded by

$$
\lambda_{i t}(t) \leq \dot{z}(t) \leq \lambda_{f t}(t), \kappa_{i t}(t) \leq z(t) \leq \kappa_{f t}(t),
$$

where $\lambda_{i t}(t)$ and $\kappa_{i t}(t)$ are some functions with respect to $t$ that exist for infinite time. Therefore, a solution of the zero dynamics with initial conditions $\left|\dot{z}_{0}\right|>\sqrt{D_{\gamma} / d_{\beta}}$ is explosively unstable and the solution escapes to positive infinity in finite time. This completes the proof.

Remark 4: The opposite of Theorem 3 is equally true when $0<d_{\beta} \leq \beta(z) \leq D_{\beta}$ for every $z \in \mathbb{R}$. $\triangleleft$ 


\section{F. Internal Dynamics Analysis}

This section shows that Theorem 1 and Theorem 3 can be extended to the internal dynamics analysis, a nonlinear unobservable dynamics when constraint of output zeroing control is not satisfied yet such as $y \neq 0$, that is $\theta_{2} \neq \theta_{2 d}$.

Theorem 4: Consider the internal dynamics given by an ordinary differential equation

$$
\alpha(y, z) \ddot{z}+\beta(y, z) \dot{z}^{2}+\gamma(y, z)=\delta(y, \dot{y})
$$

where $0<d_{\alpha} \leq \alpha(y, z) \leq D_{\alpha}$ and $|\gamma(y, z)| \leq D_{\gamma}$ for every $y, z \in \mathbb{R}$. Moreover, $\delta(y, \dot{y})$ is a function with respect to $y, \dot{y} \in \mathbb{R}$. Suppose that $D_{\delta}, d_{\delta}>0$ are positive constants which bound $-d_{\delta} \leq \delta(y, \dot{y}) \leq D_{\delta}$. If $-D_{\beta} \leq \beta(y, z) \leq-d_{\beta}<0$ for every $y, z \in \mathbb{R}$, then a solution $z(t)$ with initial conditions $\left|\dot{z}_{0}\right|>\sqrt{\left(D_{\gamma}+D_{\delta}\right) / d_{\beta}}$ is explosively unstable and the solution escapes to positive infinity in finite time.

Proof: Substituting the differential equation given by (66) into $|\gamma(y, z)| \leq D_{\gamma}$ gives

$$
\frac{d_{\beta} \dot{z}^{2}-\left(D_{\gamma}+D_{\delta}\right)}{D_{\alpha}} \leq \ddot{z} \leq \frac{D_{\beta} \dot{z}^{2}+\left(d_{\gamma}+d_{\delta}\right)}{d_{\alpha}} .
$$

The rest of this proof is omitted since similar calculations in Theorem 3 can be applied. Difference between Theorem 3 and Theorem 4 is the conservativeness.

Remark 5: The opposite of Theorem 4 is equally true when $0<d_{\beta} \leq \beta(y, z) \leq D_{\beta}$ for every $y, z \in \mathbb{R}$. $\triangleleft$

Remark 6: The internal dynamics of the Pendubot is given by

$$
\alpha(y) \ddot{\theta}_{1}+\beta(y) \dot{\theta}_{1}^{2}+\gamma\left(y, \theta_{1}\right)=p_{3}\left(K_{P} y+K_{D} \dot{y}\right),
$$

which can be rewritten as

$$
\delta(y, \dot{y})=p_{3}\left(K_{P} y+K_{D} \dot{y}\right)
$$

when constraint of output zeroing control is not satisfied yet as $y \neq 0$, that is $\theta_{2} \neq \theta_{2 d}$.

\section{Numerical Simulations}

This section performs numerical simulations to verify the effectiveness of the proposed control strategy. For the numerical validation, this section is divided into four parts with different initial conditions. The first and second sections show the performance of the designed controller with initial conditions chosen to satisfy the constraint $y=0$, that is $\theta_{2}=\theta_{2 d}$. The third and fourth sections show the desired and undesired effects of the internal dynamics with initial

TABLE I

PHYSICAL PARAMETER OF THE PENDUBOT.

\begin{tabular}{c|c|c||c|c|c}
\hline Par. & Units & Value & Par. & Units & Value \\
\hline \hline$m_{1}$ & $\mathrm{~kg}$ & 1.00 & $m_{2}$ & $\mathrm{~kg}$ & 1.50 \\
$l_{1}$ & $\mathrm{~m}$ & 0.20 & $l_{2}$ & $\mathrm{~m}$ & 0.30 \\
$l_{g 1}$ & $\mathrm{~m}$ & $l_{1} / 2$ & $l_{g 2}$ & $\mathrm{~m}$ & $l_{2} / 2$ \\
$J_{1}$ & $\mathrm{kgm}^{2}$ & $m_{1} l_{1}^{2} / 12$ & $J_{2}$ & $\mathrm{kgm}^{2}$ & $m_{2} l_{2}^{2} / 12$ \\
$m_{b}$ & $\mathrm{~kg}$ & 0.05 & $g$ & $\mathrm{~m} / \mathrm{s}^{2}$ & 9.81 \\
\hline
\end{tabular}

conditions not to satisfy the constraint, namely $y \neq 0$, that is $\theta_{2} \neq \theta_{2 d}$. Although initial conditions change over sections, feedback gains which converge the external dynamics exponentially to zero are fixed as $K_{P}=200$ and $K_{D}=30$. The desired angle of joint 2 is also designed as $\theta_{2 d}=-\pi / 2$. The end-effector releases the ball when it enters the first quadrant for the first time in a positive rotation as mentioned in Section II.

\section{A. Control via Unstable Zero Dynamics}

This section deals with numerical simulations for the initial condition chosen to satisfy the constraint $y=0$, that is $\theta_{2}=\theta_{2 d}$, to verify the validity of the designed controller based on the exact analysis of explosively unstable zero dynamics. Initial condition, thus, is chosen as $x_{0}=$ $[-0.59,-1.57,0,0]^{T}$, which places the ball directly below joint 1 when the angle of joint 2 is initialized as $\theta_{20}=-\pi / 2$.

Snapshots for dynamic acceleration of the ball driven by the explosively unstable zero dynamics are shown in Fig. 3. For the instability analysis in the previous section, the zero dynamics exhibits the explosive instability if initial condition of the zero dynamics, namely $\left(\theta_{10}, \dot{\theta}_{10}\right)$, lies outside of the maximum amplitude of periodic motion, emphasized by red lines in Fig. 2. The phase plane of the external and internal dynamics is shown in Fig. 5(a). The red line denotes the divergence of the internal dynamics in a positive rotation while the blue line denotes the convergence of the external dynamics driven by output zeroing control. Fig. 5(b) shows the time behavior of the external dynamics which is constrained to remain identically zero. Fig. 5(b) also guarantees $L_{g} h(x) \neq 0$ which implies that the exhibition of finite escape time caused by the explosive instability is independent of singular points in the input transformation of the preliminary feedback. The time behaviors of joint angles and angular velocities are shown in Fig. 5(c) and Fig. 5(d), respectively. These figures show that the designed controller successfully achieves dynamic acceleration of the ball with the initial condition.

\section{B. Control via Periodic Zero Dynamics}

This section deals with numerical simulations for initial conditions chosen to satisfy the constraint $y=0$, that is $\theta_{2}=$ $\theta_{2 d}$, to show periodic motion of the zero dynamics based on the exact analysis. Initial conditions, thus, are chosen as $x_{0}=[-0.40,-1.57,0,0]^{T}$, which casually places the ball at a certain point when the angle of joint 1 is initialized as $\theta_{10}=0$.

Snapshots for periodic motion of the Pendubot driven by the orbitally stable zero dynamics are shown in Fig. 4. For the instability analysis in the previous section, the zero dynamics exhibits the periodic stability if initial conditions of the zero dynamics, namely $\left(\theta_{10}, \dot{\theta}_{10}\right)$, lie inside of the maximum amplitude of periodic motion, emphasized by red lines in Fig. 2. The phase plane of the external and internal dynamics is shown in Fig. 6(a). The red line denotes the convergence of the internal dynamics to a periodic orbit while the blue line denotes the convergence of the external dynamics driven by 


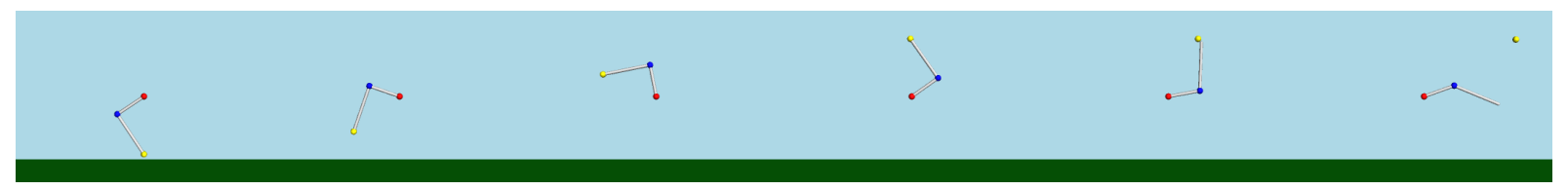

Fig. 3. Snapshots of throwing motion control via the unstable zero dynamics.

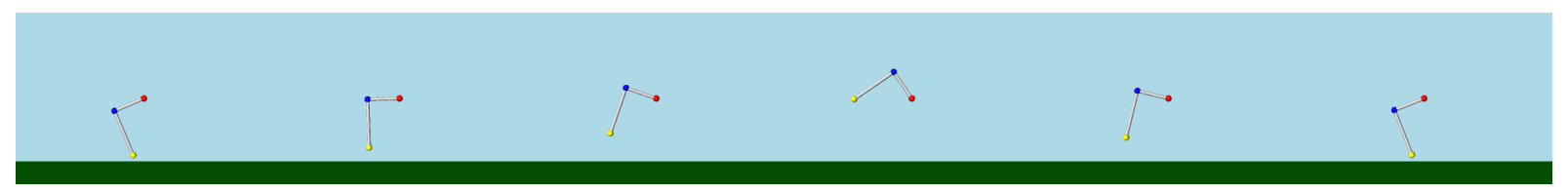

Fig. 4. Snapshots of throwing motion control for the periodic zero dynamics.

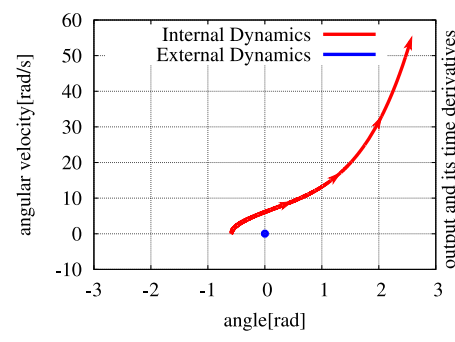

(a) phase plane

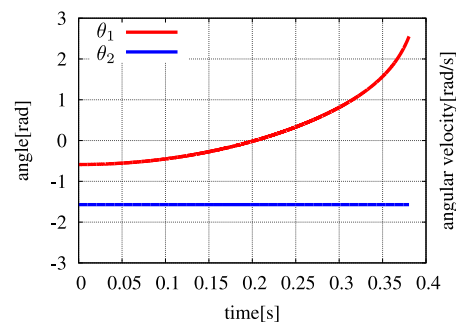

(c) joint angles

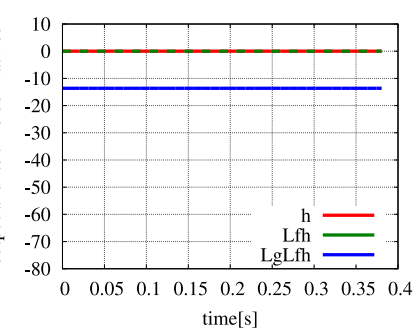

(b) external dynamics

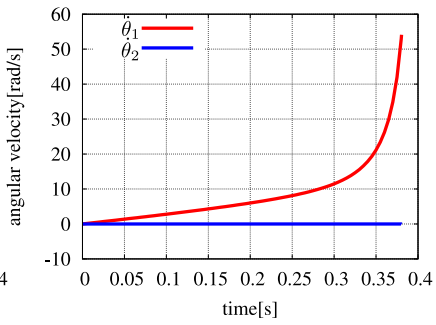

(d) angular velocities

Fig. 5. Results for output zeroing control via the unstable zero dynamics.

output zeroing control. Fig. 6(b) shows the time behavior of the external dynamics which is constrained to remain identically zero. Fig. 6(b) also guarantees $L_{g} h(x) \neq 0$ which implies that the exhibition of finite escape time caused by the periodic stability is independent of singular points in the input transformation of the preliminary feedback. The time behaviors of joint angles and angular velocities are shown in Fig. 6(c) and Fig. 6(d), respectively. These figures show that the designed controller causes periodic motion of the Pendubot with the initial conditions above.

\section{CONCLUSION}

This paper dealt with throwing motion control of the Pendubot. The controller was designed based on input-output linearization and output zeroing control. The zero dynamics was intentionally destabilized to achieve dynamic acceleration of the ball and was analytically derived to guarantee the explosive instability. Numerical simulations confirmed the validity of the designed controller based on the instability analysis of the zero dynamics.

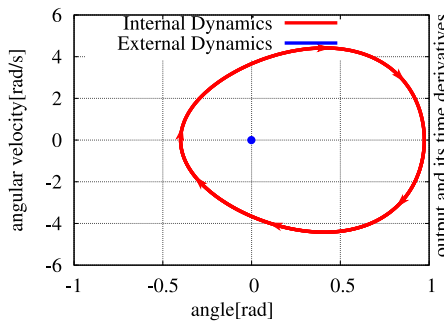

(a) phase plane

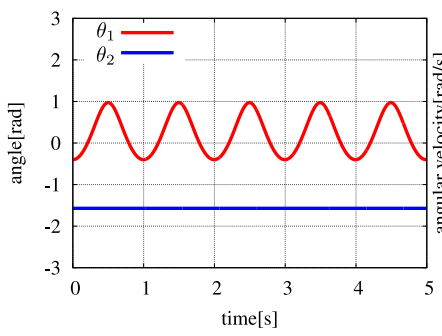

(c) joint angles

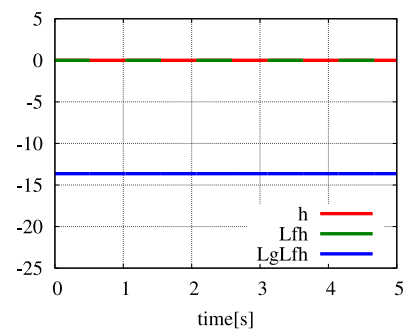

(b) external dynamics

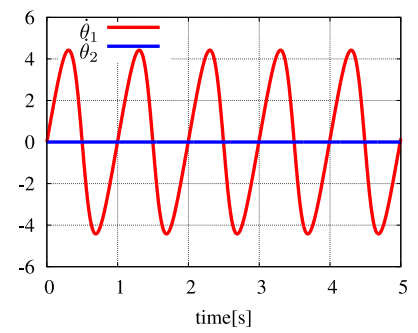

(d) angular velocities
Fig. 6. Results for output zeroing control via the periodic zero dynamics.

\section{REFERENCES}

[1] E. R. Westervelt, J. W. Grizzle, C. Chevallereau, J. H. Choi, and B. Morris, Feedback Control of Dynamic Bipedal Robot Locomotion, 1st ed., CRC Press, 2007.

[2] A. Shiriaev, J. W. Perram, and C. Canudas-de-Wit, "Constructive Tool For Orbital Stabilization of Underactuated Nonlinear Systems: Virtual Constraints Approach," in IEEE Trans. Autom. Control, vol. 50, no. 8, pp. 1164-1176, 2005.

[3] L. Freidovich, A. Robertsson, A. Shiriaev, and R. Johansson, "Periodic Motions of the Pendubot via Virtual Holonomic Constraints: Theory and Experiments," in Automatica, vol. 44, no. 3, pp. 785-791, 2008.

[4] U. Mettin, A. Shiriaev, L. Freidovich, and M. Sampei, "Optimal Ball Pitching with an Underactuated Model of a Human Arm," in Proc. IEEE Int. Conf. Robotics Automation, Anchorage, AK, USA, May. 2010, pp. 5009-5014.

[5] S. Katsumata, S. Ichinose, T. Shoji, S. Nakaura, and M. Sampei, "Throwing Motion Control based on Output Zeroing utilizing 2-Link Underactuated Arm," in Proc. Amer. Control Conf., St. Louis, MO, USA, Jun. 2009, pp. 3057-3064.

[6] S. Ichinose, S. Katsumata, S. Nakaura, and M. Sampei, "Throwing Motion Control Experiment utilizing 2-Link Arm with Passive Joint," in Proc. SICE Annu. Conf., Chofu, Japan, Aug. 2008, pp. 3256-3261.

[7] T. Shoji, S. Nakaura, and M. Sampei, "Throwing Motion Control of the Springed Pendubot via Unstable Zero Dynamics," in Proc. IEEE Multi-Conf. Syst. Control, Yokohama, Japan, Sep. 2010, pp. 16021607. 